\title{
From Catalyst Coated Membranes to Porous Transport Electrode Based Configurations in PEM Water Electrolyzers
}

\author{
Melanie Bühler, ${ }^{1,2, z}$ Peter Holzapfel, ${ }^{3}$ David McLaughlin, ${ }^{3}$ and Simon Thiele $\oplus^{3,4, z}$ \\ ${ }^{1}$ Hahn-Schickard, 79110 Freiburg, Germany \\ ${ }_{2}^{2}$ Institute for Microsystems Engineering, Laboratory for MEMS applications, Albert-Ludwigs-University of Freiburg, \\ 79110 Freiburg, Germany \\ ${ }^{3}$ Forschungszentrum Jülich GmbH, Helmholtz-Institute Erlangen-Nürnberg for Renewable Energy (IEK-11), 91058 \\ Erlangen, Germany \\ ${ }^{4}$ Department of Chemical and Biological Engineering, Friedrich-Alexander-Universität Erlangen-Nürnberg, 91058 \\ Erlangen, Germany
}

\begin{abstract}
So far the superior cell polarization behavior of membrane electrode assemblies (MEAs) using catalyst coated membranes (CCMs) as compared to those using porous transport electrodes (PTEs) was a paradigm in proton exchange membrane water electrolyzers (PEMWEs). However, this paradigm was so far neither systematically investigated nor understood. In this study, we investigate the changes in PEMWE polarization behavior upon gradually changing the MEA from a full CCM toward a full PTE-type configuration. We explain all observed findings based on the idea for a structural model of discontinuous catalyst layers. Our results show, that for current densities above $750 \mathrm{~mA} \mathrm{~cm}^{-2}$, PTE-based MEAs can result in a better polarization behavior than CCMs. Therefore, the prevailing paradigm was disproved. CCMs showed better kinetics, while PTE-type configurations performed more reproducible than CCMs despite rougher surfaces. Due to the trend of a stabilizing HFR-free cell voltage, an improved mass transport behavior of the PTE-type configurations at high current densities is assumed. Within the error-tolerance, no clear differences between PTE and CCM-based configurations in ohmic resistance could be determined. We conclude that PTE-based configurations for PEMWE, as alternatives to standard CCM-configurations, could be highly important for future manufacturing techniques depending on the application's needs.

(C) The Author(s) 2019. Published by ECS. This is an open access article distributed under the terms of the Creative Commons Attribution 4.0 License (CC BY, http://creativecommons.org/licenses/by/4.0/), which permits unrestricted reuse of the work in any medium, provided the original work is properly cited. [DOI: 10.1149/2.0581914jes]

(cc) BY
\end{abstract}

Manuscript submitted May 29, 2019; revised manuscript received August 21, 2019. Published September 27, 2019.

Proton exchange membrane water electrolyzers (PEMWEs) are considered a central technology for a future hydrogen-based economy. ${ }^{1}$ The most important element for PEMWE operation is the membrane electrode assembly (MEA). The MEA consists of anode and cathode catalyst layers and a solid polymer electrolyte membrane in between. There is no doubt that the right choice of materials for PEMWE MEAs is essential to improve lifetime, performance and cost. So far this fact resulted in a carbon supported platinum catalyst for the cathode, pristine or titanium supported iridium catalyst for the anode and Nafion both as proton exchange membrane (PEM) material and as additive for the catalyst layers. ${ }^{2}$ The standard MEA-configuration is a catalyst coated membrane (CCM), with both catalyst layers deposited onto the membrane. ${ }^{3}$ To enable a multiphase transport to and from the catalyst layers, porous transport layers (PTLs) are pressed against the CCM from both sides. For the anode side, porous titanium substrates are used to meet the durability necessities of PEMWE operation conditions. Titanium PTLs come in many different forms, with fiber or powder sintered structures being the base case. ${ }^{4}$ On the cathode side, typically carbon-based PTLs are used. These PTLs are already widely studied for PEM fuel cell applications (e.g. ${ }^{5}$ ). CCMs are manufactured either by directly depositing catalyst layers on the membrane or via the DECAL process. ${ }^{6}$ The latter consists in using Teflon blanks as a transfer substrate, on which the catalyst layers are manufactured on in a first step, and then hot-pressed on the PEM in a second step. The DECAL process is usually applied, because direct spraying on the membrane can result in membrane deformation due to the wetting behavior of solvents in the deposited ink. Alternative manufacturing routes for PEMWE MEAs, other than the above-described CCM approach, are seldom investigated.

One other MEA-configuration that appears sporadically is the PEM sandwiched between porous transport electrodes (PTEs), which are the PTLs coated directly with the catalyst layers. Grigoriev et al. ${ }^{7}$ tested different porous sintered titanium substrates when spray coated with an Ir-based catalyst layer compared to the PTLs pressed against the CCM. The worse performance of the PTE-based MEA-configurations

zE-mail: melanie.buehler@hahn-schickard.de; si.thiele@fz-juelich.de was explained by the reduced direct protonic interface between catalyst layer and membrane. Choe et al. introduced a PTE with the iridium catalyst electrodeposited on a titanium mesh PTL. Therefore, an intermediate contact between catalyst particles and PTL was reached which increased the mass activity. ${ }^{8}$ The reason for an increased mass activity when using PTEs was shown by Kang et al. ${ }^{9}$ for the cathode side via visualization of hydrogen bubble formation at the platinum based catalyst layer. For this, a structured thin titanium foil with circular, straight pores was pressed against a reference CCM and compared with the approach of sputter coating the titanium foil directly with the platinum catalyst. For both configurations, hydrogen bubbles were only visible at the edges of the pores of the thin titanium foil and not in the area in between. This indicates that large parts of the catalyst layers in the CCM-configuration are inactive, when not in direct contact with the PTL. Increasing the catalyst mass activity is therefore one motivation for the development of PTE-based configurations, but also the possibility to substitute costly titanium substrates at the anode side. A stable PEMWE operation was shown e.g. for carbon paper coated either directly with the iridium-based catalyst or first with a titanium-based adhesive intermediate layer. ${ }^{10-12}$ Besides high performance and low costs one major criteria for the applicability in commercial PEMWE is the long term stability. It is known from literature, that the single materials used in a PEMWE cell are prone to different degradation mechanisms which influence the polarization behavior and affect the mechanical stability. ${ }^{13-15}$ When changing interfacial properties, as well as the microstructure of the catalyst layers, the long term behavior would most probably change for every MEA-configuration tested in this work. PTE-configurations could be advantageous compared to CCM-based systems, since coating the titanium PTL directly with the catalyst layer could establish a corrosion protection layer for the titanium substrate ${ }^{8}$ and ensure a stable electronic interface between catalyst particles and the PTL. The long term stability of the PTE-based configurations was however not tested in the frame of this work but is a crucial quality factor when searching for the best MEA-configuration for commercial applications.

All works on PTE-based configurations are first steps toward high performing PTE-configurations, but the reasons for a changed polarization behavior compared to CCM-configurations, have so far 
never been more deeply studied and understood. This study therefore investigates for the first time, the influence on the polarization behavior of a PEMWE, when the electrode-membrane interface of a PEMWE MEA was changed gradually from a CCM toward a cathodic PTE (cPTE) and anodic PTE (aPTE) configuration. In literature, there are modifications of the membrane-electrode interface in a PEMWE CCM setup such as roughening the membrane ${ }^{16}$ or introducing an additional Nafion layer ${ }^{17}$ on top of the PEM before depositing the catalyst layers. Consequently, in this work we also investigate a thin Nafion impregnation of the aPTE, with the expectation of an improved protonic interface.

A simple idea for a structural model was used to explain the influence of changed catalyst layer structures and interfaces on the polarization behavior. The model is based on three general cases connecting the size scales of catalyst particles with the pore space of different supports: (i) If the pore sizes of a substrate are smaller or in the range of the catalyst particle size, the catalyst layer will be homogeneous. (ii) If the pores are larger than the catalyst particles, the porous support is most likely infiltrated by the catalyst ink during deposition. This would result in a continuous, but rougher catalyst layer. (iii) If the pores are larger than the catalyst particles and additionally larger than the thickness of the catalyst layer, discontinuities of the catalyst layer can occur. In this work, we elucidate which of these cases are relevant, when changing from pure CCM to full PTE-based PEMWE MEA-configurations.

\section{Experimental}

Membrane electrode assembly._Different MEA-configurations were tested in this work with a focus on systematically changing the membrane-electrode interfaces. To minimize the influence of catalyst and membrane materials as well as of different fabrication methods, materials purchased from the same manufacturer were used for all experiments. CCMs and half-sided CCMs with only the anode or cathode catalyst layer deposited on a Nafion N117 membrane were purchased from FuelCellsEtc. The samples had a catalyst loading of $1.5 \mathrm{mg} \mathrm{cm}^{-2} \mathrm{IrO}_{2}$ for the anode side and $0.5 \mathrm{mg} \mathrm{cm}^{-2} \mathrm{PtC}(60 \%)$ on the cathode side. A carbon cloth material (FuelCellsEtc, type GDLCT) was chosen as cathode PTL (cPTL) and a sintered titanium fiber substrate (Bekaert, $1 \mathrm{~mm}$ thick, 57\% porosity, $20 \mu \mathrm{m}$ fiber diameter) was used as anodic PTL (aPTL). Free-standing cathode porous transport electrodes (cPTE) with a loading of $0.5 \mathrm{mg} \mathrm{cm}^{-2} \mathrm{PtC}(60 \%)$ were commercially available based on the GLD-CT (FuelCellsEtc, type SLGDE). The carbon substrates and commercial cPTEs were punched out and the titanium PTLs laser cut into $5 \mathrm{~cm}^{2}$ squares to meet the same active areas as of the full and half-sided CCMs. Free-standing anodic porous transport electrodes (aPTEs) were not commercially available, and were therefore manufactured in-house. For this purpose, an $\mathrm{IrO}_{2}$-based catalyst layer was spray coated on top of the Bekaert titanium fiber substrates as described below. To eliminate influences on the polarization behavior due to different fabrication parameters, a second reference $\mathrm{CCM} *$ was prepared for studying the anode side of the different MEA-configurations. The CCM* was a commercial half-sided cathode CCM (FuelCellsEtc, $0.5 \mathrm{mg} \mathrm{cm}^{-2} \mathrm{PtC}(60 \%)$ on Nafion N117) spray coated with the same anode catalyst ink as used for preparing the aPTEs.

The $\mathrm{IrO}_{2}$ ink for spray coating the anodic catalyst layers consisted of $1 \mathrm{wt} \%$ solids and $99 \mathrm{wt} \%$ solvents (DI-water and 2-Propanol in equal parts). The solids consisted of $98 \mathrm{wt} \% \mathrm{IrO}_{2}$ Premion (Alfa Aesar) and $2 \mathrm{wt} \%$ Nafion D520 (FuelCellStore). The catalyst powder was weighed in a glass bottle and then wetted by the appropriate amount of DI-water. Afterwards 2-Propanol followed by Nafion was added. The ink for spray coating needs to be homogeneously mixed. To this end, the bottle was gently shaken after adding each new component. The final ink was continuously stirred and sonicated for $30 \mathrm{~min}$ (Hielscher, model UIS250L, $0.55 \mathrm{~W}, 90 \%$ amplitude) in an ice bath. The ink was then placed on a magnetic stirrer overnight and the $30 \mathrm{~min}$ mixing procedure was repeated right before spray coating. A spray coater (Sono-Tek, model Exacta Coat) was used with an ultrasonic nozzle type AccuMist
(48 kHz). The following parameters were used for spray coating: path speed of $170 \mathrm{~mm} / \mathrm{sec}$, shaping air of $0.6 \mathrm{kPa}$, hot plate temperature of $120^{\circ} \mathrm{C}, 5 \mathrm{~W}$ ultrasonic power and flow rate of $0.45 \mathrm{ml} / \mathrm{min}$. The spray pattern was meander shaped with a pitch of $0.75 \mathrm{~mm}$. The nozzle height was set to $37 \mathrm{~mm}$. To monitor the noble metal loading during spray coating, a $1 \mathrm{~cm}^{2}$ rectangular metal piece was spray coated as well and weighed on a microscale (Sartorius, model ME 36S). To deposit the anode catalyst layers, the porous titanium substrates, as well as the half-sided cathode CCMs were fixed in PTFE frames. The exact amount of Nafion in the final self-made anodic catalyst layers, and based on that also the noble metal loading, was determined via thermogravimetrical analysis (TGA) (Netsch, model STA 449F5) according to the burning characteristics of Nafion. ${ }^{18}$ Powder for the TGA analysis was collected via scratching the catalyst layer off the PTFE frames. The collected sample was heated under air atmosphere at a rate of $5 \mathrm{~K} / \mathrm{min}$ up to $1000^{\circ} \mathrm{C}$ (hold for one hour). A Nafion content of ca. $9 \mathrm{wt} \%$ and a noble metal loading of $1.4 \mathrm{mg} \mathrm{cm}^{-2} \mathrm{IrO}_{2}$ was determined. The difference between the as-mixed and final Nafion content could be a result of catalyst particle sedimentation along the tubing of the spray coater as well as in the syringe.

Since the cPTEs were commercially manufactured with a thin Nafion coating to improve the water management and adhesion toward the membrane, the same was tested for the aPTEs. Therefore, impregnated aPTEs (ai PTEs) were prepared by spray coating a thin Nafion layer (2.5 wt\% D2020 in 2-Propanol) on the surface of the aPTEs. Spray parameters were: $90 \mathrm{~mm} / \mathrm{s}$ path speed (meander shaped, $0.5 \mathrm{~mm}$ pitch), flow rate of $0.2 \mathrm{ml} / \mathrm{min}, 2.5 \mathrm{~W}$ ultrasonic power, $0.6 \mathrm{kPa}$ shaping air, $37 \mathrm{~mm}$ nozzle height. The aPTEs were either placed on a hot plate at $65^{\circ} \mathrm{C}$ or at $120^{\circ} \mathrm{C}$ during spray coating. The average thickness of the final Nafion coating was calculated from Raman analysis to be approximately $1 \pm 0.2 \mu \mathrm{m}$.

Structural characterization.-Surfaces and cross-sections were analyzed in terms of porosity, thickness and homogeneity. To this end, images were taken using a focused ion beam (FIB) scanning electron microscope (SEM) (Zeiss, model Crossbeam 540 with GEMINI II). To analyze the distribution of Nafion at the surface of the ai PTEs as well as along the cross-sections, an EDX mapping was performed (Oxford Instruments, model X-Max 150 using Aztec software).

To determine the thickness of the additional Nafion layer on the aPTEs, a glass slide was coated with 36 layers of Nafion and then analyzed with a confocal Raman microscope (WITec, model alpha 300). The Raman microscope was equipped with a $532 \mathrm{~nm}$ laser, operated at $50 \mathrm{~mW}$. The microscope was coupled to a WITec UHTS VIS-NIR spectrometer with a $300 \mathrm{gr} / \mathrm{mm}$ optical grating and a thermoelectrically cooled CCD-camera for acquiring Raman spectra. A metallurgical objective (Zeiss, model LD EC Epiplan-Neofluar 50x/0.55) was used to operate the microscope. A confocal depth scan at a step size of $500 \mathrm{~nm}$ through the membrane was performed. After a background subtraction (WITec Project FIVE+), the summarized intensity of the $v_{\mathrm{s}}(\mathrm{C}-\mathrm{F})$ mode ( 700 to $760 \mathrm{~cm}^{-1}$ ) was used for a segmentation of the membrane within the line scan using Otsu's method in Matlab (MathWorks). The thickness was calculated from the segmented data and corrected for spherical aberration, induced by the refractive index mismatch between Nafion $\left(\mathrm{n}_{\text {Nafion }}=1.38^{19}\right)$ and air $\left(\mathrm{n}_{\text {air }}=1\right)$, by multiplying the obtained value with $\mathrm{n}_{\text {Nafion }} / \mathrm{n}_{\text {air }}{ }^{20}$ A single point measurement of 36 layers of Nafion on the glass slide revealed a thickness of around $7.6 \pm 0.9 \mu \mathrm{m}$

Electrochemical characterization.-The different MEAconfigurations were tested at atmospheric pressure in a self-designed test cell setup. The outermost parts of the test cell were aluminum plates tightened with eight screws at $8.5 \mathrm{Nm}$ to hold the inner cell parts. Two heating elements, set to $80^{\circ} \mathrm{C}$, were inserted into the Al plates. The inner cell parts were electrically connected to a potentiostat (Scribner, model 857) via two copper plates at the anode and cathode side respectively. The copper plates were electrically insulated on the side facing the aluminum plates via a self-adhesive PTFE foil. On the other side, titanium flow fields were in direct 
contact with the copper plates. The flow fields both at the anode and cathode side were made of titanium plates with a $5 \mathrm{~cm}^{2}$ milled parallel finger structure to evenly distribute water toward the PTLs. The PTLs, were made of fiber sintered porous titanium at the anode and carbon cloth at the cathode. The PTLs had the same geometric area as the finger structure of the flow field. PTFE frames were used to align the PTLs and also acted as hard-stops to control the assembly pressure of the cell. The PTFE frame at the anode side was as thick as the titanium PTL (1 mm) to avoid a "step" between PTFE frame and hard titanium substrate toward the MEA. At the cathode side the PTFE frame was thinner $(150 \mu \mathrm{m})$ than the carbon PTL $(410 \mu \mathrm{m})$ to set a sufficient compression. The MEA was then sandwiched between the PTLs. Two metal pins were used to align the different materials and were removed before testing the cell. The internal cell resistance without membrane was calculated to ca. $0.091 \Omega \mathrm{cm}^{2}$ from a set current and the corresponding cell voltage (repeated three times). To be able to reduce this high resistance, it is planned in future work to apply a conductive gold coating on top of the titanium flow fields to further reduce parasitic contact resistances.

Anode and cathode were supplied separately with DI-water via a peristaltic pump (Ismatec, model IP 65) at a flow rate of $40 \mathrm{ml} / \mathrm{min}$. The tubing close to the inlets of the test cell was immersed into a water-filled thermostat set to $88^{\circ} \mathrm{C}$ (Lauda, model Ecoline 003) to prevent temperature gradients in the cell. Once a stable cell temperature of $80^{\circ} \mathrm{C}$ was observed (monitored continuously with a temperature sensor), the test cell was conditioned in potentiostatic mode from $1.4 \mathrm{~V}-2.2 \mathrm{~V}$ in $200 \mathrm{mV}$ steps. Each voltage step was held for $30 \mathrm{~s}$ and the complete range was scanned for 15 times. Afterwards polarization curves were recorded as follows: $0 \mathrm{~A}-0.04 \mathrm{~A}$ in $10 \mathrm{~mA}$ steps, $0.05 \mathrm{~A}-1 \mathrm{~A}$ in $50 \mathrm{~mA}$ steps, $1.25 \mathrm{~A}-3 \mathrm{~A}$ in $250 \mathrm{~mA}$ steps, $3.5 \mathrm{~A}-5 \mathrm{~A}$ in $500 \mathrm{~mA}$ steps and above $6 \mathrm{~A}$ in $1 \mathrm{~A}$ steps for the CCM-configurations and in $500 \mathrm{~mA}$ steps to record more data points of the lesser-known PTE-configurations. Every step was held for $120 \mathrm{~s}$.

The high frequency resistance (HFR) was measured via the software "FlowCell" (Scribner) at a frequency of $1 \mathrm{kHz}$. The measured HFR was then applied to the experimental polarization curves for an analysis of the HFR-free cell voltage. The polarization data was analyzed in terms of contributions to the kinetic, ohmic and mass transport overpotential. ${ }^{21}$ Three samples per MEA-configuration were prepared and tested. The average values and standard deviation therefore gives an impression of the reproducibility of the respective MEAconfigurations. To substantiate the gained knowledge regarding separate contributions of the anode and cathode side on the polarization behavior in changed MEA-configurations, the use of a reference electrode (e.g. ${ }^{10}$ ) was suggested. In future works such an extension would be beneficial.

\section{Results and Discussion}

Design of experimental and research hypothesis.-The aim of this study was to understand in depth what changes occur in an MEA upon changing from a CCM-type configuration toward a PTE-type configuration, with the electrodes deposited directly onto the PTLs. The leading questions in this study were: Is there a benefit when using PTE-type configurations? What are advantages and disadvantages of the different configurations? Are there potentials for a future change in the manufacturing process, which at the moment concentrates on CCM-based approaches? All this was discussed based on systematic and reproduced measurements, in which a gradual transition from a CCM-type configuration to a full PTE-type configuration (Figure 1) was studied. To avoid unwanted cross dependencies of different fabrication techniques and catalyst materials, a second reference $\mathrm{CCM} *$ was prepared (Figure $1 \mathrm{C}$ ) for a direct comparison with the MEA-configurations using an aPTE. The anode side of the $\mathrm{CCM} *$-configuration consisted of the same anode catalyst layer and was prepared with the same manufacturing method (spray coating) and with the same parameters as used for preparing the anodes in the PTE-configurations. At the cathode side, the material composition and fabrication technique was constant for all MEAconfigurations, since all materials could be purchased from the same supplier.

We investigated all six different MEA-configurations in the following in terms of polarization curves and a breakdown analysis of kinetic contributions, ohmic resistance and mass transport. Complementarily, we analyzed the structure of the samples using methods based on electron microscopic methods. Based on these considerations we used a simple structural model (Figure 2) to clarify our expectations for the influence of the different MEA-configurations tested in this work (Figure 1). When the catalyst layers are directly deposited onto flat membranes as in the CCM-configurations (Figure 1A,1C), protonic transport is most likely improved, due to the geometrically shortest possible protonic pathway between all anode and cathode catalyst particles for a given PEM thickness. At the same time, the electrically conductive interface facing toward the PTLs could be suboptimal, since the PTLs are only mechanically pressed against the CCM. Therefore, at the anode side, only a few titanium fibers establish a direct electric contact between catalyst layer and PTL (Figure 2A). Regarding mass transport, the distribution of water and gases within the catalyst layer could be less than optimal, since all transport must be performed through a compact catalyst layer. Mass transport through the pristine, uncoated PTLs pressed against the CCM however, is supposed to be optimal.

When applying the anode catalyst layer directly on the titanium PTL as in the aPTE-configuration (Figure 1D), the deposition of large

MEA configurations tested in this work

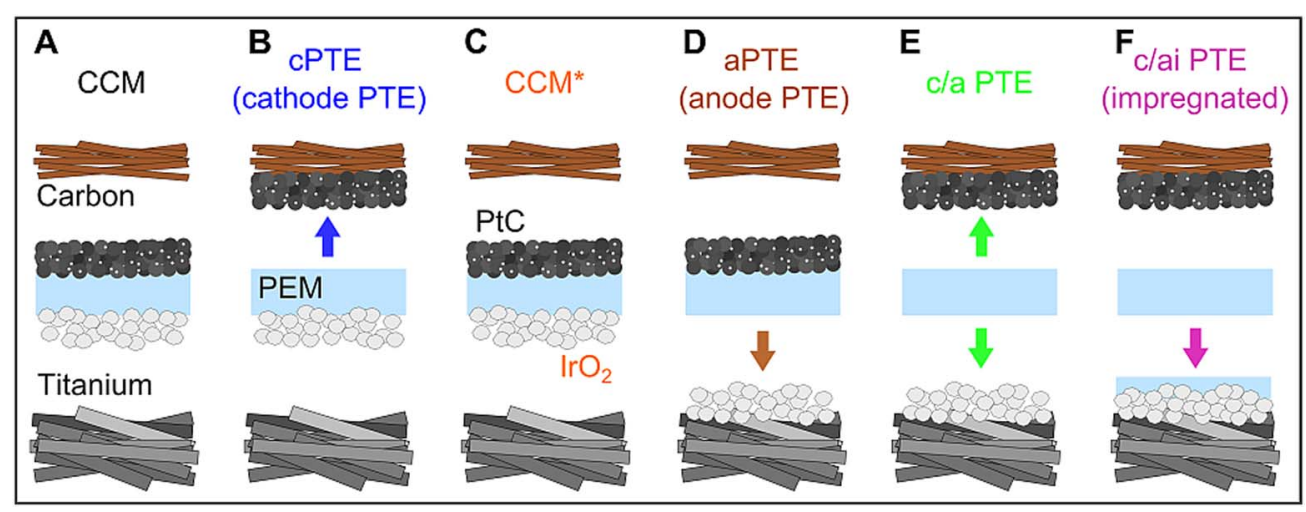

Figure 1. A Commercial catalyst coated membrane (CCM) with both electrodes deposited on the membrane B Commercial catalyst coated cathode porous transport electrode (cPTE) C CCM with in-house manufactured anode catalyst layer deposited on a commercial half-sided cathode CCM denoted CCM $*$ D Catalyst coated anode porous transport electrode (aPTE) produced with the same in-house manufactured anode as used for CCM* E Combined aPTE- and cPTE-configuration with a bare membrane in between denoted as c/a PTE F cPTE and Nafion impregnated aPTE named as c/ai PTE. 

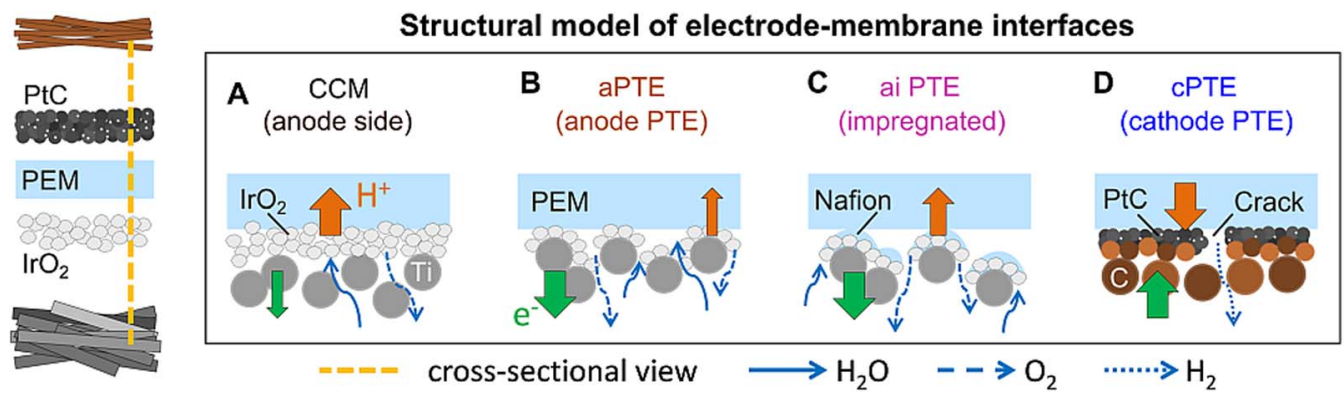

Figure 2. Model of the membrane-electrode interfaces and transport processes in different MEA-configurations. Thinner arrows imply a relative decrease of a certain transport process. A Anode side of a CCM pressed against titanium fibers B Anode-PEM interface facing toward a freestanding aPTE C Interface between PEM and impregnated ai PTE D Cathode-PEM interface when a cPTE is used.

parts of the catalyst layer up to multiple hundreds of micrometers into the pores of the titanium PTL (Figure 2B) is expected. Grigoriev et al. discussed, that in this case fewer parts of the catalyst layer are in direct contact with the solid membrane. ${ }^{7}$ Therefore, first the direct protonic interface between catalyst layer and the PEM is reduced, which would result in an increased ohmic resistance. Moreover, when some catalyst particles are disconnected from the protonic network within the catalyst layers, a lower catalyst utilization and therefore comparably worse kinetics are most likely to result. Depositing the catalyst particles directly on the titanium fibers however, improves the electric interface compared to the CCM-configuration. The rough, porous electrode surface could improve mass transport, but the risk of blocked pores due to the comparably deeper infiltration of the aPTL during deposition of the catalyst material exists. This infiltration would then increase mass transport problems. For the cPTEs (Figure 1B), a much more planar carbon-based support compared to the titanium substrates is used. Therefore, one would assume less poorly connected catalyst areas (Figure 2D). Cracks in the micro porous layer (MPL) could however lead to similar effects as in the aPTE case. Consequently, worse kinetics, better mass transport and counteracting protonic vs. electric resistance could appear. For the c/ai PTE (Figure 1F) we expected a behavior based on the aPTE and cPTE base cases, but an improvement of the ionic transport and consequently an improved HFR (Figure 2C).

Influence of cathode PTE (cPTE) on the polarization behavior.The influence of the position of the cathode catalyst layer on the polarization behavior was analyzed by comparing the performance of commercial CCMs with a commercial cPTE-configuration (Figure 3A). Both configurations were purchased from the same supplier, using the same loading of catalysts as well as the same manufacturing method (compare methods section). One polarization curve each of three different samples per configuration were analyzed. The size of the single standard deviation ( $\sim 68 \%$ confidence interval), characterized the reproducibility of the considered MEA-configuration (Figure 3B). At
A

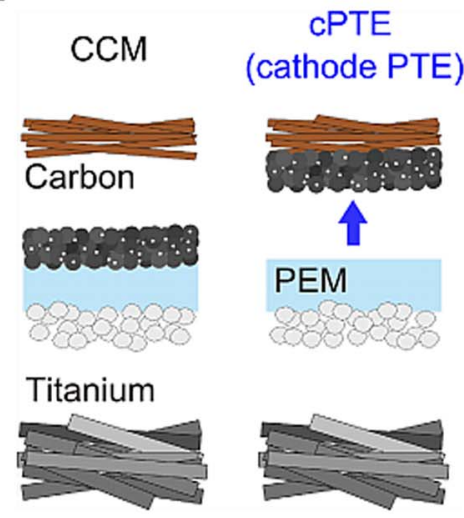

B

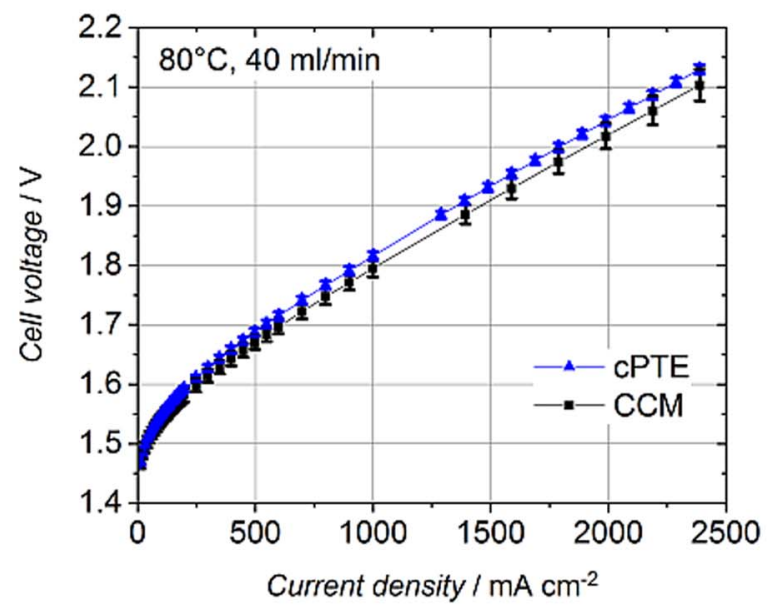

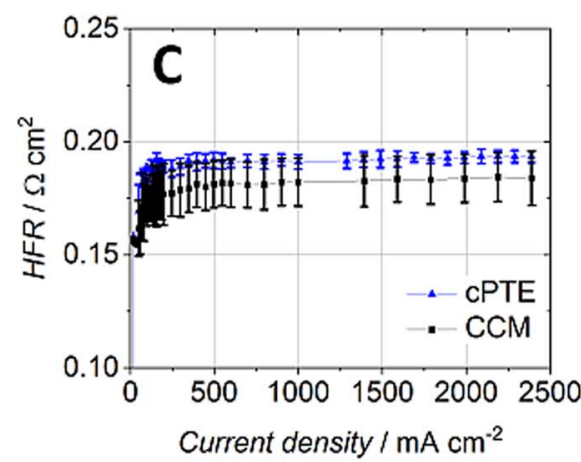
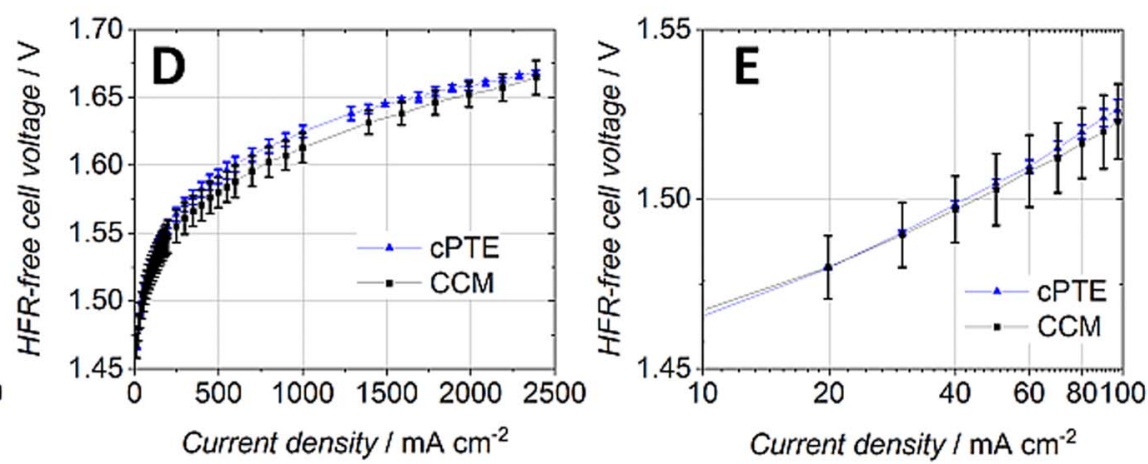

Figure 3. A Schematic of the tested MEA-configurations CCM vs. cPTE B Polarization curves C High frequency resistances D HFR-free cell voltage E HFR-free cell voltage at low current densities. 

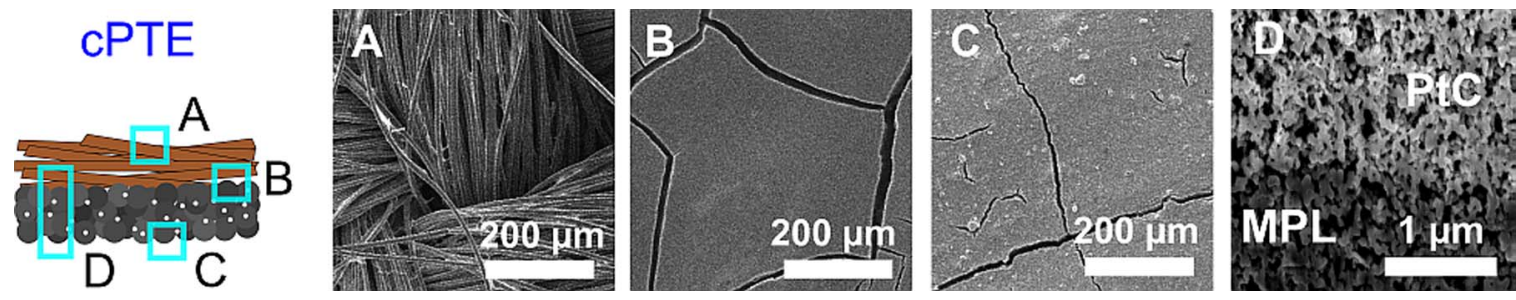

Figure 4. A Backside of the carbon cloth porous transport layer B Microporous layer (MPL) deposited on the carbon cloth material facing the electrode C PtC catalyst layer deposited on the microporous layer forming the final cPTE D Cross-section of the cPTE showing the MPL and PtC catalyst layer.

the transition between $5 \mathrm{~A}$ and the last segment of the polarization curve starting at $6 \mathrm{~A}$, unfortunately an inaccurate recording of the cell voltage at $1.2 \mathrm{~A} \mathrm{~cm}^{-2}(6 \mathrm{~A})$ occurred for some samples of the CCM- and cPTE-configurations. This data points could not be used to calculate the average and standard deviation and are not shown in Figure 3.

Experimental results.-Full CCMs performed slightly better than cPTEs (Figure 3B), due to lower ohmic losses (Figure 3C) and barely better due to a better HFR-free cell voltage (Figure 3D). The kinetic region at low current densities was very similar for both configurations (Figure 3E). Interestingly, the standard deviation of the cPTE polarization curves was significantly smaller than of the full CCM. Particularly at higher current densities, the full CCM showed a less reproducible operation than the cPTEs (Figure 3B).

Structural analysis.-SEM images and a FIB-SEM cross-section analysis confirmed, that the carbon-based cPTLs (Figure 4A) provided a comparably flat surface for depositing the cathode catalyst layer on top (Figure 4C), due to the MPL (Figure 4B). The cross-section showed the smooth interface between MPL and cathode catalyst layer (Figure 4D) of the cPTE. Consequently, there was no strong variation of the membrane-electrode interface between CCM- and cPTE-configuration. Some cracks were found throughout the MPL (Figure 4B). These cracks are less significant for the cPTE.

Discussion of the results.-Due to the relatively smooth surfaces of both the carbon PTL as well as the cPTE, the loss of catalyst material in the cracks of the carbon materials was negligible. Therefore, no larger disconnected 'catalyst islands' and consequently no significant differences in the kinetic region were observed. In accordance with the above-discussed qualitative structural model (Figure 2), we found slightly higher mass transport losses for the cPTEs. This might be since smaller cracks in the cPTE surface allowed for less water and gas distribution, compared to a cPTL with bigger cracks in the MPL being pressed onto the CCM. Coating the PTLs with catalyst material therefore reduces the pore size at the interface toward the catalyst layers, which was then most probably a hindrance for mass transportation through the electrodes. The slightly higher Tafel slope of the cPTE-configuration (Figure 3E) is as well an indication, that mass transport losses are more pronounced, when the PTL is coated with the catalyst material. Trinke et al. recently analyzed the correlation between cathode electrode structure and mass transport overpotential. ${ }^{22}$ They found an increase of ionomer content within the cathode catalyst layer to cause higher mass transport resistances. The removal of hydrogen from the catalyst layer was impeded with increased ionomer contents, which is supposed to be due to a decreased pore space and longer transport paths within the ionomer. Consequently, this leads to high dissolved hydrogen concentrations at the interface between membrane and electrode. This supersaturation of dissolved hydrogen was then indicated as a reason for a higher gas crossover through the PEM and higher mass transport losses due to higher cathode half-cell potentials. For the cPTE-configuration tested in this work nevertheless, at higher current densities, the difference of the HFR-free voltage (kinetic and mass transport overpotential) between cPTE and CCM-configurations became smaller. One explanation could be a beneficial mass transport of the cPTEs at higher current densities, but also other contributions e.g. of the effective proton resistance within the catalyst layers have to be considered. The HFR as the combination of ionic and electric resistances was either equally good or slightly worse for the cPTE compared to the CCM. This was not fully derivable from the HFR data due to the significantly higher variation of the CCM-configuration than compared to the cPTEconfiguration. Reasons for the lower reproducibility of the CCMs were difficult to assess, as this also depends on the process parameters chosen for the production. One possible explanation would be the intermediate electronic interface between catalyst layer and PTLs in the PTEconfigurations due to the direct deposition of the catalyst layers on the titanium and carbon substrates. In the CCM-configuration, bending of the membrane during assembly or operation of the membrane could lead to a changed number of direct contact points between CCM and PTLs which would then lead to e.g. different HFRs within the same MEA-configuration.

Influence of anode PTE (aPTE) and symmetric PTE (c/a PTE) on the polarization behavior.-In this section a spray coated aPTE with the anodic catalyst layer deposited directly on the porous titanium substrate was compared to a reference $\mathrm{CCM} *$ with the same anodic catalyst layer deposited on a half-sided cathodic CCM (Figure 5A). In the combined c/a PTE-configuration, a membrane was sandwiched between the cPTE and aPTE. All experiments were performed at least three times with a single standard deviation $(\sim 68 \%$ confidence interval) representing the reproducibility of the investigated MEA-configuration.

Experimental results.-As can be derived from Figure 5B, full $\mathrm{CCM} * \mathrm{~s}$ performed slightly better at lower current densities (below $\sim 750 \mathrm{~mA} \mathrm{~cm}^{-2}$ ) while both aPTE and c/a PTE performed better and were more reproducible at higher current densities (above $\sim 750 \mathrm{~mA} \mathrm{~cm}{ }^{-2}$ ). An analysis of the different contributions to the polarization curve revealed the reasons for this behavior: The kinetic losses can be derived from a semi-logarithmic plot of the HFRfree cell voltage (Figure 5E) at low current densities. The CCM*configuration showed the lowest kinetic overpotential, whereas the aPTE and c/a PTE-configurations were shifted to a higher value. With increasing current density, contributions of the mass transport overpotential became more and more pronounced as it was visible in the HFRfree cell voltage over the whole current density range (Figure 5D). The $\mathrm{CCM} *$-configuration showed the best HFR-free cell voltage, but with increasing current density the difference between the three configurations became less significant and variations between the different $\mathrm{CCM} *$-configurations became more pronounced. The reason for these trends should be further analyzed especially when aiming for PEMWE cells operating at higher current densities and when planning to apply pressure to the system. Suermann et al. ${ }^{23}$ e.g. showed, that with increasing operating pressure, the mass transport overpotential is reduced. Therefore, the difference between the three CCM* samples could have a minor impact on the reproducibility at higher operating pressures compared to atmospheric conditions in this work. The main reason for the worse overall performance of the $\mathrm{CCM} *$-configuration 
A

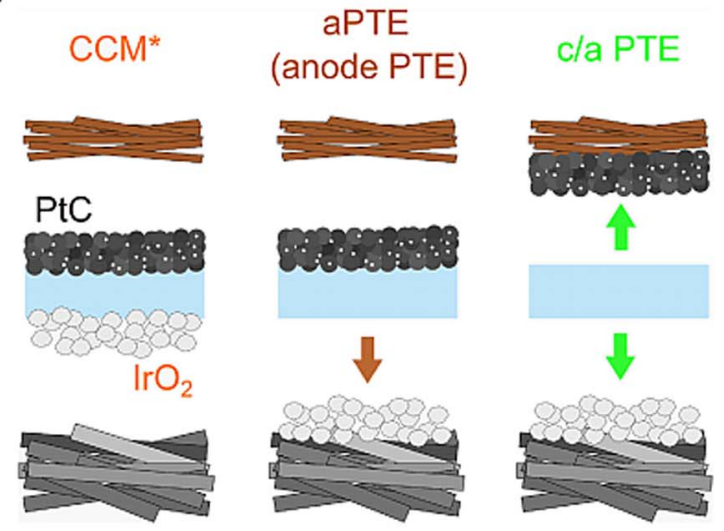

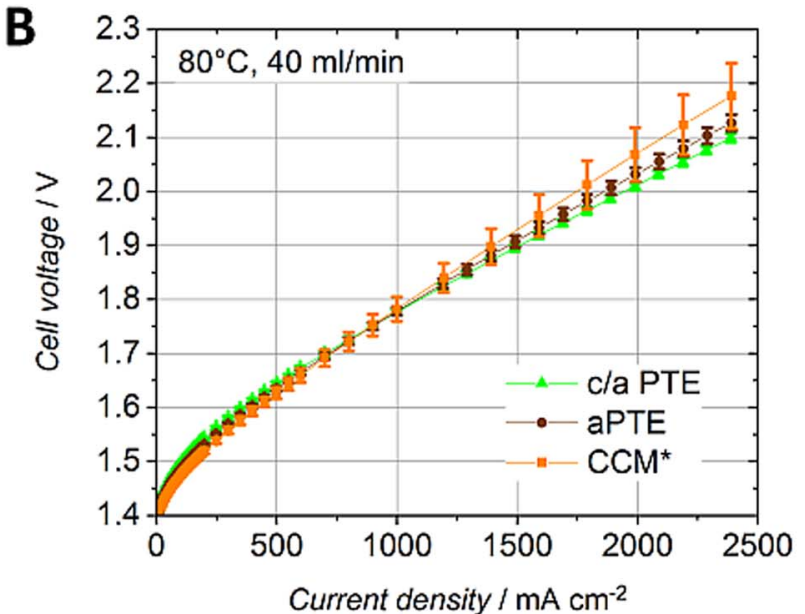
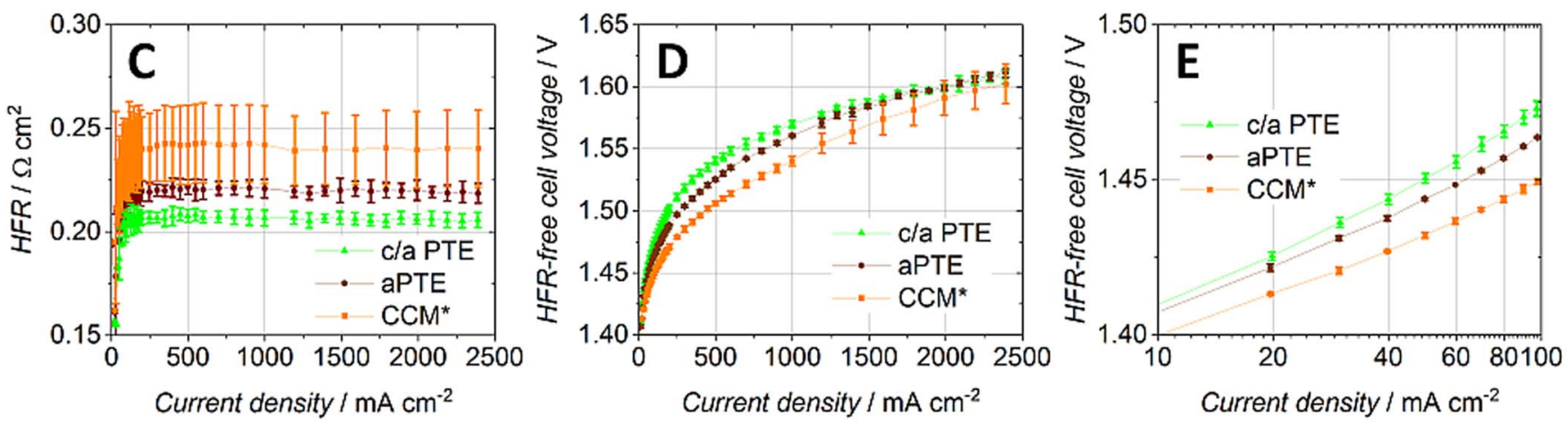

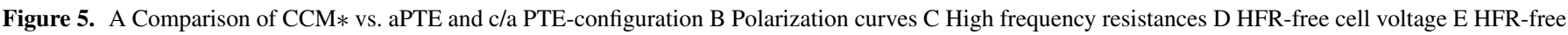
cell voltage at low current densities.

at higher current densities was a higher average HFR (Figure 5C), which was significantly less reproducible than the HFR of the aPTEand c/a PTE-configurations.

Structural analysis.-As expected, SEM images showed a rough surface of the titanium-based PTLs (Figure 6A). In contrast to carbon-based PTLs no titanium PTL with MPL is commercially available. Consequently, the interface between membrane and anode was significantly changed when depositing the anode catalyst layer on top of the porous titanium substrate compared to a flat membrane. In the aPTE-configuration (Figure 6B), parts of the anode catalyst layer were deposited deep into the pores of the aPTL. Therefore, parts of the catalyst material are placed in great distance from the membrane. At the large scale, the catalyst layer of the aPTE showed an inhomogeneous distribution of the catalyst particles on top of the titanium fibers (Figure 6C). However, as can be derived from the cross-section (Figure 6D), the deposited layer itself is homogeneous.
Discussion of the results.-According to the idea of a structural electrode model discussed above (Figure 2), we had the case of a highly inhomogeneous electrode structure for the aPTEs. The resulting disconnected 'catalyst islands' were expected to result in worse kinetics, which was clearly the case (Figure 5E). When advancing from an aPTE to a c/a PTE, we observed only a slight increase of kinetic overpotential, which was expected according to the results when varying the cathode side in the previous section. The CCM*configuration showed the lowest HFR-free cell voltage (Figure 5D), but is approaching the PTE-based configurations at higher current densities. As discussed in the methods section, a beneficial mass transport behavior of PTE-configurations at high current densities compared to CCMs could be one explanation, but also e.g. the effective proton resistance has to be considered, as analyzed by Babic et al. for anodes in CCM-configurations. ${ }^{24}$ When considering the HFR behavior however, the situation became more complicated: on the one hand, the ionic conductivity was expected to be worse in the aPTE and c/a PTE-configurations compared to the $\mathrm{CCM} *$, on the other hand,
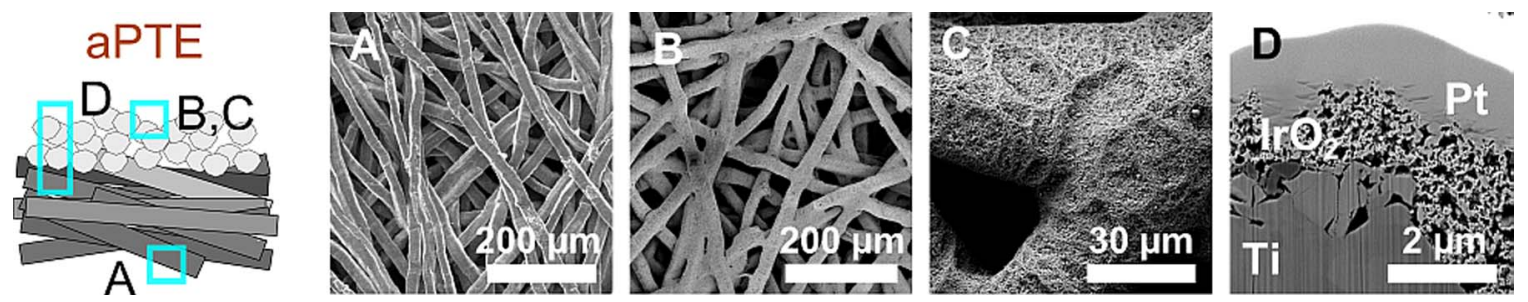

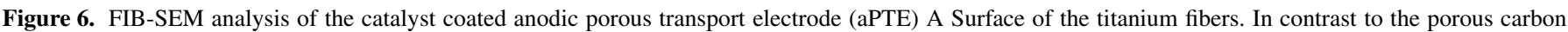

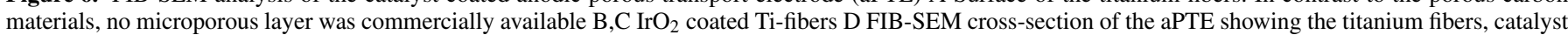
layer and Pt protection layer (deposited during the FIB-SEM imaging process). 
A

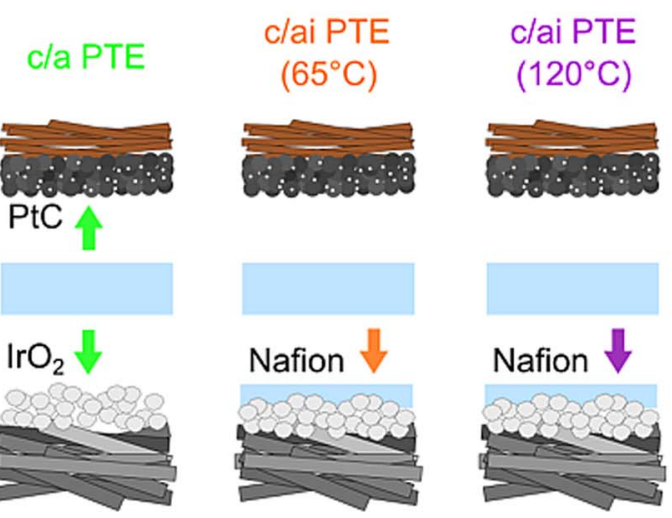

B

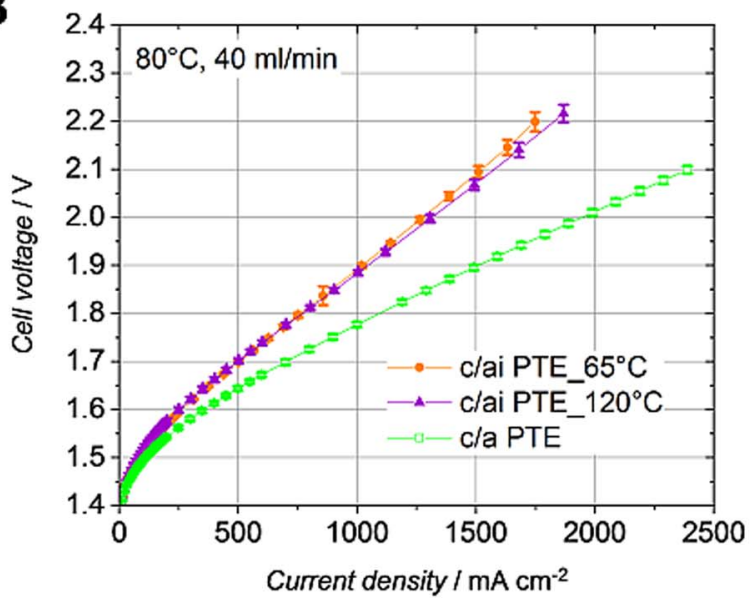

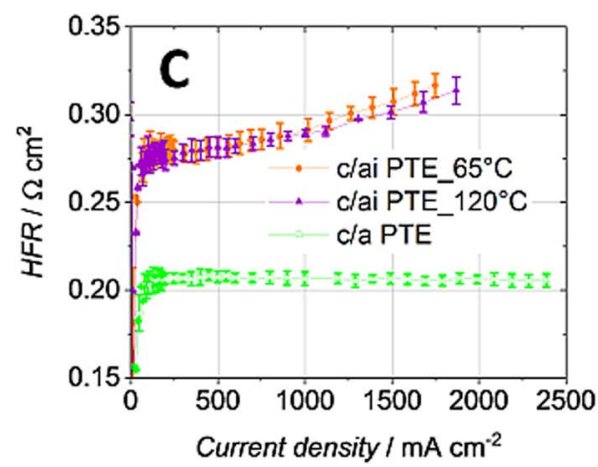
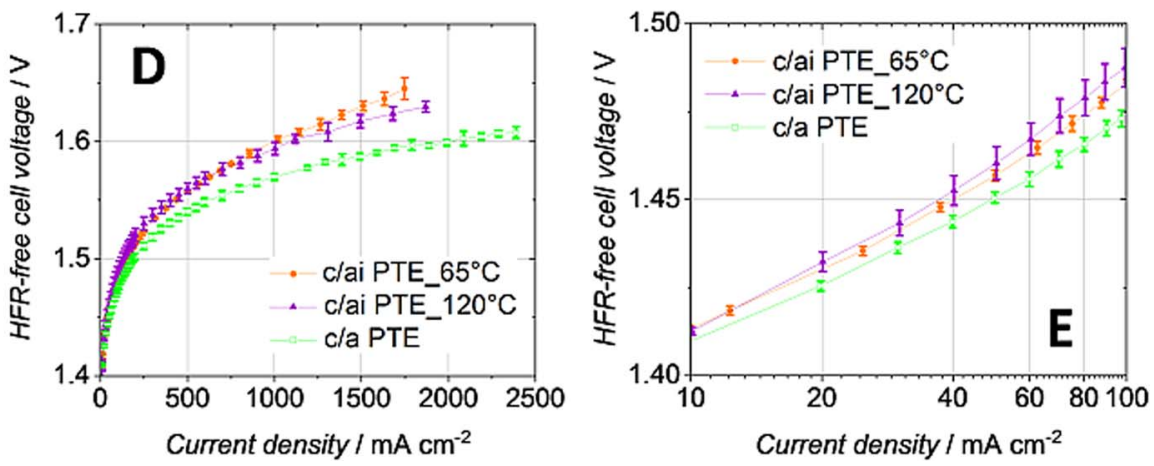

Figure 7. A Configurations c/a PTE vs. c/ai PTE (fabricated at $65^{\circ} \mathrm{C}$ and $120^{\circ} \mathrm{C}$ ) B Polarization curves C High frequency resistances D HFR-free cell voltage E HFR-free cell voltage at low current densities.

improvements in electric conductivity were expected, due to a better electrical interface contact. In Figure $5 \mathrm{C}$ we observed that the $\mathrm{CCM} *$ exhibited a worse average HFR $\left(0.24 \Omega \mathrm{cm}^{2}\right)$ than the aPTE and c/a PTE-configurations. This HFR value was unexpected when compared to the value of the CCM from Figure 3C of which was $0.18 \Omega \mathrm{cm}^{2}$. We assume that our $\mathrm{CCM} *$ manufacturing process could be further optimized with respect to ionic contact. Here also a hotpressing step might be advantageous, but would have altered the catalyst layer structure compared to the aPTEs. This was to be avoided, as we concentrated on only structural layer effects in this study. Apart from that, we observed what we expected from the discussion of our model: the interfacial contact area between the catalyst layer and the membrane when using the aPTE-configuration was smaller compared to the cPTE-configuration with a flat carbon substrate. Therefore, the HFR of the aPTE-configuration was around $0.22 \Omega \mathrm{cm}^{2}$ (Figure 5C) compared to the cPTE-configuration with a HFR of around $0.19 \Omega \mathrm{cm}^{2}$ (Figure 3C). This explained, why, for the c/a PTE-configuration, as a combination of the aPTE- and cPTE-configurations, we observed a decreased HFR of $0.21 \Omega \mathrm{cm}^{2}$ compared to the aPTE (Figure 5C). Also in this study, we observed, that the reproducibility was highest for the c/a PTE-configuration, a bit lower for the aPTE-configuration and the $\mathrm{CCM} *$ approach was found to have the worst reproducibility. The same effect was seen in the previous section when comparing the CCM with the cPTE polarization behavior. In summary, we saw a tendency in both studies that there was an improvement of the reproducibility when changing from a CCM-configuration to a PTE-configuration.

Influence of Nafion impregnated anode PTE (ai PTE) on the polarization behavior.-In this section, we investigated the influence of an additional Nafion D2020 layer deposited directly on the aPTE. The basic idea was that this kind of an approach could improve the ionic interface resistance between electrode and membrane. At higher tem- peratures of the aPTEs, the additional Nafion layer was expected to dry immediately at the aPTEs surface whereas at lower temperatures a certain degree of infiltration into the pores of the aPTE was expected. Therefore, two different types of ai PTEs were fabricated at a temperature of $65^{\circ} \mathrm{C}$ as well as $120^{\circ} \mathrm{C}$. The c/ai PTE-configurations were then compared to the c/a PTE-configuration presented in the previous section (Figure 7A). Three samples per configuration were prepared and tested (Figure 7B).

Experimental results.-Impregnating the aPTE with Nafion strongly reduced the overall cell performance (Figure 7B). One reason was an increased HFR of the c/ai PTE-configurations with a strong dependency on the current density (Figure 7C). In both c/ai PTEconfigurations mass transport losses were obvious when analyzing the HFR-free cell voltage (Figure 7D). The c/ai PTE prepared at $65^{\circ} \mathrm{C}$ showed a slightly stronger increase of mass transport overpotential with current density than the c/ai PTE prepared at higher temperature. The HFR-free cell voltage at low current densities (Figure 7E) showed similar kinetics for the c/ai PTE-configurations, which were both slightly worse than the c/a PTE-configuration. The reproducibility of the c/ai PTE prepared at $120^{\circ} \mathrm{C}$ was slightly worse in the kinetic region than for the other configurations.

Structural analysis. - The surface of the ai PTE impregnated at $120^{\circ} \mathrm{C}$ (Figure 8A) was analyzed with an EDX tool to map the fluorine content at the surface which showed large, inhomogeneous fields of Nafion covering the catalyst layer (Figure 8B). The cross-section revealed a continuous Nafion coating (Figure $8 \mathrm{C}$ ). Therefore, voids between the Nafion coating and the catalyst layer were observed. A fluorine mapping of the cross-section showed the continuous character of the impregnation at $120^{\circ} \mathrm{C}$ (Figure $8 \mathrm{D}$ ) on the local scale. However, the samples showed a strong variation of the global thickness of the 

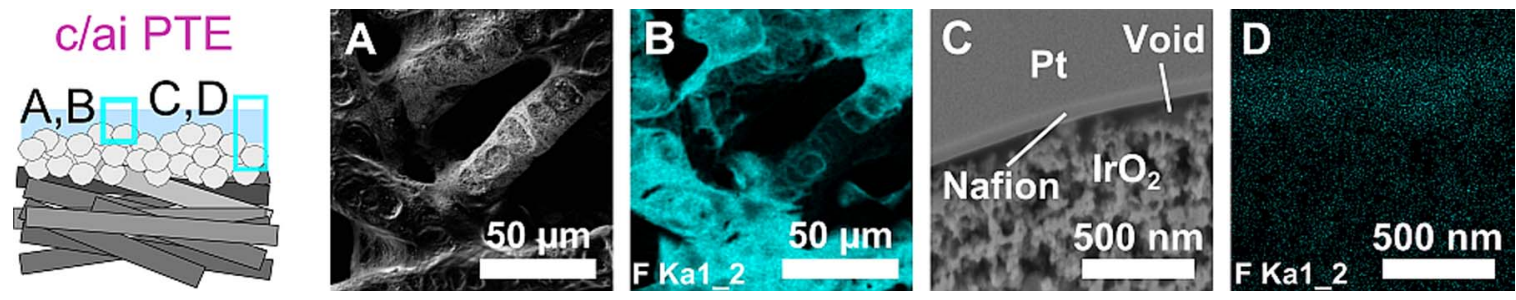

Figure 8. A Surface of the ai PTE coated at $120^{\circ} \mathrm{C}$ B EDX mapping of $\mathrm{F}$ of the impregnated surface C FIB-SEM cross-section of the ai $120^{\circ} \mathrm{C}$ PTE with protective Pt layer showing voids between the Nafion coating and the catalyst layer D EDX of the cross-section showing the Nafion coating on top of the aPTE (C and D do not show the same position).

Nafion coating, first due to the rough surface of the aPTE itself and second most probably due to an inhomogeneous drying of the Nafion layer.

Discussion of the results.-For the c/ai PTE, we expected a behavior based on the c/a PTE-configuration, but with an improvement of the ionic transport and consequently a reduced HFR. However, the experimental results were not in line with this hypothesis: We not only found a higher initial HFR for the c/ai PTEs, but also discovered a worse mass transport. More interestingly, the additional Nafion impregnation caused an increase in HFR as a function of current density, indicating worse ionic transport and interfacial contact resistances. We used the following model to explain changes of the polarization behavior when a c/ai PTE-configuration impregnated at different temperatures (high $\mathrm{T}$ vs. low $\mathrm{T}$ ) is used (Figure 9):

At high temperatures, solvents are expected to dry immediately at the surface of the aPTE (Figure 9, high T). The fast drying of solvents prevents the deep infiltration of the pores of the catalyst layer with Nafion. Therefore, a continuous Nafion layer as seen in the crosssection of the c/ai PTE coated at $120^{\circ} \mathrm{C}$ (Figure $8 \mathrm{C}$ ) is most probably the case. Voids between the Nafion layer and the catalyst layer increase the protonic interfacial contact resistance and therefore the HFR. When coated at low temperatures (Figure 9, low T), the additional Nafion layer is expected to infiltrate the pores of the aPTE to a further extend. This would lead to an intermediate contact of the surface of the aPTE with the Nafion layer and less voids on the one hand. On the other hand, the pores of the catalyst layer might be blocked, so that a higher mass transport overpotential at higher current densities could be the case. We assume, that the Nafion coating on top of the catalyst layer in combination with the solid titanium fiber below acts as diffusion barrier for evolving gas bubbles and possibly also for water. When evolving oxygen is trapped in the PTE structure, active sites might be blocked, which would increase mass transport losses. ${ }^{2}$ When the trapped gas additionally dries out parts of the Nafion phase within the catalyst layer, the proton conductivity is reduced and thus the HFR is increasing with increasing current densities.

\section{Conclusions}

In this work, we presented a detailed study on the effect on cell polarization when changing from a CCM-configuration in systematic steps to PTE configurations. PTE-based MEA-configurations showed a similar performance as CCM-based MEA-configurations and are thus suitable for PEMWE applications. Generally, PTE-type configurations showed a tendency toward worse kinetics than the CCMconfigurations. The tendency of a stabilizing HFR-free cell voltage at high current densities could be an indicator for a beneficial mass transport behavior of PTE-based configurations. The HFR evaluation was not fully conclusive due to a significantly worse reproducibility of the HFR values of the CCM-configurations compared to the PTEconfigurations. A Nafion coating did not improve the performance of the aPTEs. Instead, a Nafion coating led to a higher HFR, which further increased with current density. The Nafion coating additionally increased the mass transport overpotential. Interestingly, we found in general a strong increase in reproducibility when depositing the catalyst layers directly on the titanium and carbon substrates as in the PTE-based MEA-configurations.

We suggested a simple structural model for three different cases for porous support - catalyst layer interactions to explain the observed changes in polarization for CCM, aPTE and cPTE like structures. One key idea on the path toward understanding the polarization behavior of the analyzed MEA-configurations was the idea of disconnected 'catalyst islands' within porous supports. Based on that idea we were able to explain the differences in the contributions to the overpotential in all considered cases. By developing an idea of the structural interaction between catalyst layer, porous substrates and membrane, we intend to stimulate future improvements of the interfacial design of PEMWE MEAs.

In our study, we found that the interfaces between membrane and catalyst layer, as well as the porous transport layer have an influence on the polarization behavior of a PEMWE MEA. This highly interesting playground opens up the path toward novel MEA structures and MEA-PTL structure interactions beneficial for the PEMWE conversion efficiency. Optimizing porous transport electrodes for alternative PEMWE MEA-configurations is therefore highly promising since not only the performance can be improved. On top, also the fabrication
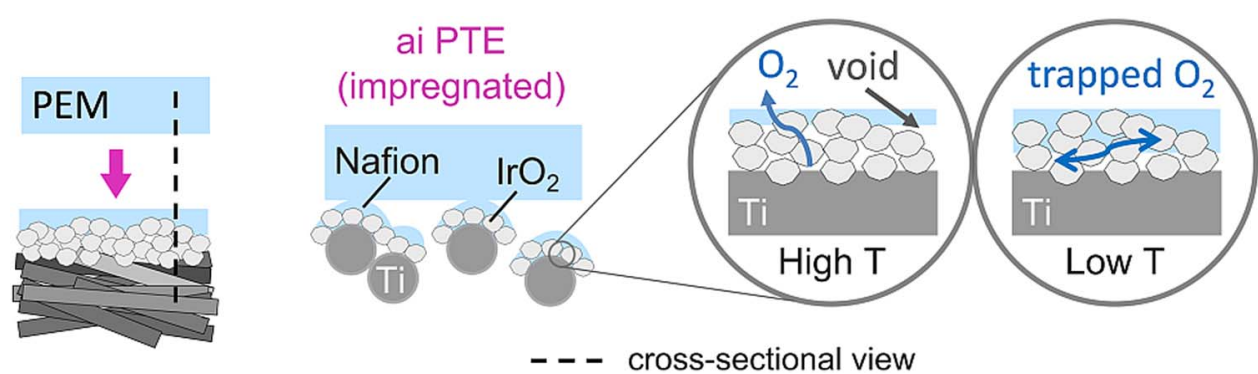

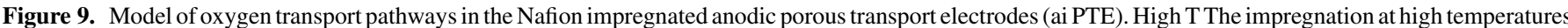

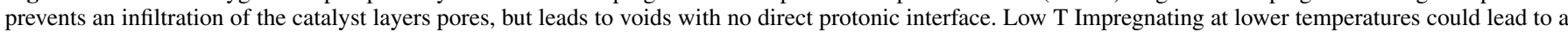
deeper infiltration of surface near pores of the catalyst layer and therefore the trapping of oxygen. 
costs could be reduced, when using a direct spray technique instead of a DECAL transfer process.

The interpretation and analysis of influences on the polarization behavior in this study were valid for a specific set of operation conditions, as well as a specific combination of material and fabrication techniques. When changing one of those parameters, the polarization behavior of the single MEA-configurations will most probably change and could therefore lead to a different picture as captured in this work. We therefore suggest to perform the presented analysis, which was a structural model idea in combination with a systematic structural and electrochemical investigation, for every new MEA-configuration at the given operation conditions. The influence of changed interfaces on the long term stability adds additional quality requirements on the way to choose the best possible MEA-configuration.

\section{Acknowledgments}

The authors gratefully acknowledge the financial support by the Federal Ministry of Education and Research of Germany in the framework of POWER-MEE (03SF0536E). We thank Michel Suermann for fruitful discussions and reviewing the paper as well as Thomas Böhm for the Raman analysis and Dunia Abed el Hafez for preparing the Nafion coatings for the c/ai PTE-configurations.

\section{ORCID}

Simon Thiele (1D https://orcid.org/0000-0002-4248-2752

\section{References}

1. A. Buttler and H. Spliethoff, Renewable Sustainable Energy Rev., 82, 2440 (2018).

2. D. G. Bessarabov, H. H. Wang, H. Li, and N. Zhao, Editors, PEM electrolysis for hydrogen production: Principles and applications, Boca Raton, CRC Press (2016)
3. U. Babic, M. Suermann, F. N. Büchi, L. Gubler, and T. J. Schmidt, J. Electrochem. Soc., 164(4), F387 (2017).

4. L. Zielke, A. Fallisch, N. Paust, R. Zengerle, and S. Thiele, RSC Adv.,4(102), 58888 (2014).

5. N. Zamel and X. Li, Prog. Energy Combust. Sci., 39(1), 111 (2013).

6. H. J. Cho, H. Jang, S. Lim, E. Cho, T.-H. Lim, I.-H. Oh, H.-J. Kim, and J. H. Jang, Int. J. Hydrogen Energy, 36(19), 12465 (2011).

7. S. A. Grigoriev, P. Millet, S. A. Volobuev, and V. N. Fateev, Int. J. Hydrogen Energy, 34(11), 4968 (2009).

8. S. Choe, B.-S. Lee, M. K. Cho, H.-J. Kim, D. Henkensmeier, S. J. Yoo, J. Y. Kim, S. Y. Lee, H. S. Park, and J. H. Jang, Applied Catalysis B: Environmental, 226, 289 (2018).

9. Z. Kang, G. Yang, J. Mo, Y. Li, S. Yu, D. A. Cullen, S. T. Retterer, T. J. Toops, G. Bender, B. S. Pivovar, J. B. Green, and F.-Y. Zhang, Nano Energy, 47, 434 (2018),

10. E. Slavcheva, I. Radev, S. Bliznakov, G. Topalov, P. Andreev, and E. Budevski, Electrochimica Acta, 52(12), 3889 (2007).

11. B.-S. Lee, S. H. Ahn, H.-Y. Park, I. Choi, S. J. Yoo, H.-J. Kim, D. Henkensmeier, J. Y. Kim, S. Park, S. W. Nam, K.-Y. Lee, and J. H. Jang, Applied Catalysis B: Environmental, 179, 285 (2015).

12. F. M. Sapountzi, S. C. Divane, E. I. Papaioannou, S. Souentie, and C. G. Vayenas, Journal of Electroanalytical Chemistry, 662(1), 116 (2011).

13. M. Suermann, B. Bensmann, and R. Hanke-Rauschenbach, J. Electrochem. Soc., 166(10), F645 (2019).

14. A. Weiß, A. Siebel, M. Bernt, T.-H. Shen, V. Tileli, and H. A. Gasteiger, J. Electrochem. Soc., 166(8), F487 (2019).

15. Q. Feng, X. Yuan, G. Liu, B. Wei, Z. Zhang, H. Li, and H. Wang, Journal of Power Sources, 366, 33 (2017).

16. Y. Zhang, C. Wang, N. Wan, Z. Liu, and Z. Mao, Electrochem. Commun., 9(4), 667 (2007).

17. S. Song, H. Zhang, B. Liu, P. Zhao, Y. Zhang, and B. Yi, Electrochem. Solid-State Lett., 10(8), B122 (2007)

18. M. Feng, R. Qu, Z. Wei, L. Wang, P. Sun, and Z. Wang, Sci. Rep., 5, 9859 (2015).

19. J. S. Santos, I. M. Raimundo, C. M. Cordeiro, C. R. Biazoli, C. A. Gouveia, and P. A. Jorge, Sens. Actuators B Chem., 196, 99 (2014).

20. S. H. Wiersma, P. Török, T. D. Visser, and P. Varga, J. Opt. Soc. Am. A, 14(7), 1482 (1997).

21. M. Suermann, T. J. Schmidt, and F. N. Büchi, Electrochim. Acta, 211, 989 (2016).

22. P. Trinke, G. P. Keeley, M. Carmo, B. Bensmann, and R. Hanke-Rauschenbach, J. Electrochem. Soc., 166(8), F465 (2019).

23. M. Suermann, K. Takanohashi, A. Lamibrac, T. J. Schmidt, and F. N. Büchi, J. Electrochem. Soc., 164(9), F973 (2017).

24. U. Babic, E. Nilsson, A. Pătru, T. J. Schmidt, and L. Gubler, J. Electrochem. Soc., 166(4), F214-F220 (2019). 